\title{
Parallel Testing Interferometer
}

\author{
James B. Saunders
}

\begin{abstract}
The conventional methods of testing the parallelism of opaque bodies, such as gage blocks, by interferometry require wringing of the body to an optical flat. This operation disturbs the temperature equilibrium, necessitating long periods between tests, especially for long blocks. It often injures the surfaces of both the optical flat and the test body. Also, if the body is a standard gage block, repeated wringings during use ultimately change the dimension. This paper describes an interferometer for measuring the parallelism of gage blocks and other bodies of any reasonable length without the necessity of the wringing operation. Two forms of this instrument are used - one for testing long blocks and another for testing short blocks. Either form can be constructed for testing blocks of any length, but two forms are found to be more practical.
\end{abstract}

\section{Introduction}

The conventional procedures for measuring the parallelism of gage blocks ${ }^{1,2}$ require the wringing of the blocks onto an optical flat. The wringing operation often injures the contacted surfaces and repeated wringings necessitate frequent refinishing of the optical flat that is used as a base. A method for measuring parallelism, without the wringing operation, has significant advantages because the danger of injury to the contacted surfaces is eliminated. Accordingly, two instruments that utilize this method are described: one is for testing very long blocks and similar bodies, whereas the other is designed for short blocks. Both instruments use low orders of interference and neither requires the use of a standard.

\section{Optics of the Interferometer}

A description of the optics for either form of this instrument covers a large portion of that for the other. For distinction we will designate them as "the long-block interferometer" and "the shortblock interferometer". The double-image prism used in these instruments is adjusted during construction ${ }^{3}$ so that a ray of light, shown in the plane of figure $1 \mathrm{~A}$, after division into two component rays, 1 and 2 , at $P_{0}$ will, on reflection at $P_{1}$ and $P_{2}$, lie in planes that are parallel to the semireflecting plane of the prism but deviate equally toward or from opposite sides of the plane of figure $1 \mathrm{~A}$. The projection of the light rays on the dividing plane is shown in figure $1 \mathrm{~B}$, which is perpendicular to the plane of figure $1 \mathrm{~A}$. This deviation is effected by rotating one component of the prism relative to the other about an axis normal to the dividing plane of the prism. If this deviation is held constant, the width of the interference fringes in the direction normal to the plane of figure $1 \mathrm{~A}$ is fixed. This component of fringe width is, therefore, frozen into the system ${ }^{4}$ when the cement between the component prisms

1 Gauges and fine measurements, by F. H. Rolt, I, p. 204 (Macmillan and Co., Ltd., London 1929)

The science of precision measurement, by The DoAll Co., p. 143 (1953)

3 Construction of a Kösters double-image prism, by J. B. Saunders, J. Research

NBS 58, 21 (1957) RP2729.

4NBS Tech. News Bul. 42, 30 (1958).
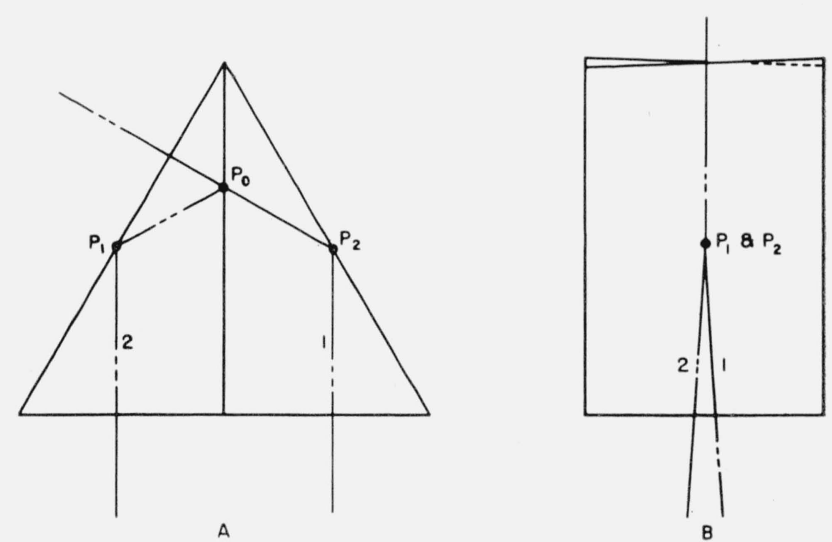

FIgURE 1. Kösters double-image prism.

A small angle is formed by the two $30^{\circ}$ edges shown exaggerated in B.

becomes hard by cooling after adjustments are complete. Furthermore, the tilting of any plane surface outside the prism that affects the two component beams between division and recombination, will produce equal effects in this direction and, consequently, will not affect the fringe width. However, the rotation of plane surfaces about an axis normal to the plane of figure $1 \mathrm{~A}$ will produce equal effects on the two component beams but in opposite directions, thus producing a proportionate effect on the fringe width in the direction parallel to this plane.

Because of the above-described properties of this prism, adjustments of the instrument in which it is used affect the fringe width in one direction only. Consequently, when measuring the parallelism of gage blocks the test can be applied to parallelism in only one direction at a time. To test for parallelism in other directions the block must be rotated.

\subsection{Long-Block Interferometer}

Figure $2 \mathrm{~A}$ is a horizontal section through the optical elements of this instrument. The light from a source at $\mathrm{S}_{1}\left(\mathrm{~S}_{2}\right)$ is collimated by lens $\mathrm{L}_{1}\left(\mathrm{~L}_{2}\right)$ and divided into two equal components by the beamdividing plane $\mathrm{B}_{1}\left(\mathrm{~B}_{2}\right)$. Each component suffers total internal reflection in the prism and emerges in planes 


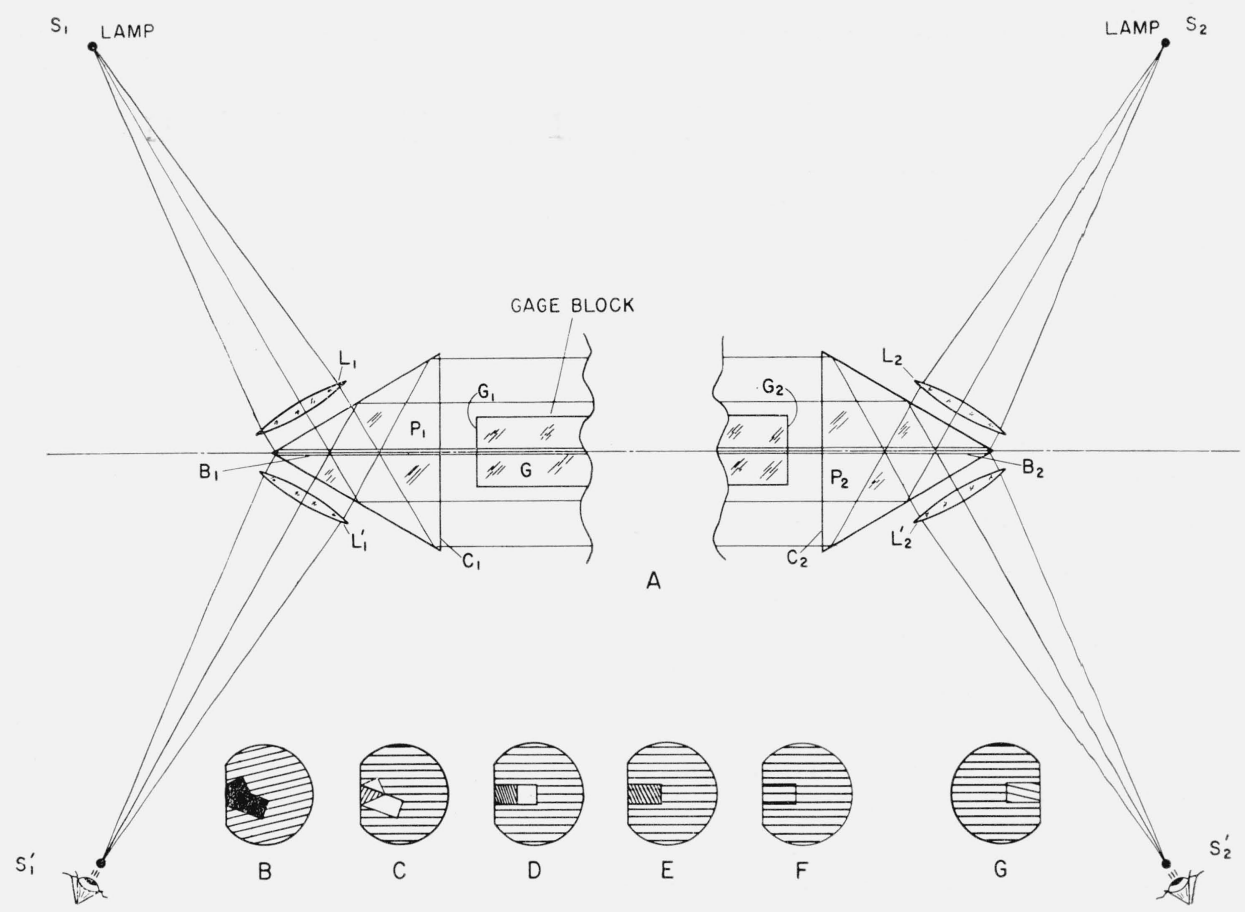

Figure 2. Optics of interferometer for testing parallelism of long blocks.

Figures $\mathrm{B}$ through $\mathrm{G}$ represent observed interference îringes.

parallel to $\mathrm{B}_{1}\left(\mathrm{~B}_{2}\right)$, but at a small angle to the base surface $\mathrm{C}_{1}\left(\mathrm{C}_{2}\right)$. The latter condition is obtained by a slight rotation of $\mathrm{P}_{1}\left(\mathrm{P}_{2}\right)$ about an axis normal to the dividing plane $\mathrm{B}_{1}\left(\mathrm{~B}_{2}\right)$. This serves to permit the elimination of light reflected from $\mathrm{C}_{1}\left(\mathrm{C}_{2}\right)$.

The two prisms, $\mathrm{P}_{1}$ and $\mathrm{P}_{2}$, are separated by a distance exceeding the length of the longest block to be tested. If desired, this distance may be made adjustable. The two dividing planes, $\mathrm{B}_{1}$ and $\mathrm{B}_{2}$, are adjusted to be coplanar and the two base faces, $\mathrm{C}_{1}$ and $\mathrm{C}_{2}$, are adjusted parallel to each other. A line joining the centers of $\mathrm{P}_{1}$ and $\mathrm{P}_{2}$ is adjusted to form a small angle with the normal to faces $\mathrm{C}_{1}$ and $\mathrm{C}_{2}$.

The light emerges from prism $\mathrm{P}_{1}\left(\mathrm{P}_{2}\right)$ as two separated components, one on each side of the dividing plane. It enters prism $\mathrm{P}_{2}\left(\mathrm{P}_{1}\right)$, again suffers total internal reflection, and each pair of component rays recombines in the plane of $\mathrm{B}_{2}\left(\mathrm{~B}_{1}\right)$. One-half of each beam proceeds to the neighborhood of source $\mathrm{S}_{2}\left(\mathrm{~S}_{1}\right)$ and the other half to $\mathrm{S}_{2}{ }^{\prime}\left(\mathrm{S}_{1}{ }^{\prime}\right)$. An observer at $\mathrm{S}_{2}{ }^{\prime}\left(\mathrm{S}_{1}{ }^{\prime}\right)$ sees a set of interference fringes that cover the entire aperture.

If a gage block, $\mathrm{G}$, is inserted in the position shown with its end face, $\mathrm{G}_{1}$, adjusted normal to the light beams, it will reflect equal and corresponding parts of the two component light beams from $\mathrm{S}_{1}$ back through $\mathrm{P}_{1}$ to $\mathrm{S}_{1}{ }^{\prime}$. Accordingly, the observer at $\mathrm{S}_{1}{ }^{\prime}$ sees a background set of fringes, produced by light from $\mathrm{S}_{2}$ and another set on the face of $\mathrm{G}_{\mathrm{i}}$, that is produced by light from $S_{1}$. Since $G_{1}$ is normal to the light beams these two sets of fringes will be parallel to each other and to the plane of figure $2 \mathrm{~A}$.
If the other end, $\mathrm{G}_{2}$, of the gage block is parallel to $\mathrm{G}_{1}$, the fringes seen at $\mathrm{S}_{2}{ }^{\prime}$ will likewise be parallel to each other and to the plane of figure $2 \mathrm{~A}$. If, however, $G_{2}$ is not parallel to $G_{1}$, in the plane of figure $2 \mathrm{~A}$, it will not be normal to the light beams, and the sets of fringes seen at $\mathrm{S}_{2}{ }^{\prime}$ will not be parallel to each other. The angle between these two sets of fringes is a measure of the angle between $G_{1}$ and $G_{2}$ in the plane of figure $2 \mathrm{~A}$. The component of the angle between $\mathrm{G}_{1}$ and $\mathrm{G}_{2}$ that is perpendicular to the plane of figure $2 \mathrm{~A}$ (or horizontally in figs. $2 \mathrm{~B}$ to $2 \mathrm{G}$ ) does not affect the fringes because it affects all pairs of component beams equally. If the component of the angle between the gage-block surfaces that is normal to the plane of figure $2 \mathrm{~A}$ (or vertically in figs. $2 \mathrm{~B}$ to $2 \mathrm{G}$ ) is desired, the block must be rotated $90^{\circ}$ and the operation repeated.

Since each prism is adjusted for complete compensation in the plane of figure $2 \mathrm{~A}$, white light can be used. A measure of the vertical width of the fringes (perpendicular to fig. 2A) for a known monochromatic light, with a micrometer eyepiece at $\mathrm{S}_{2}{ }^{\prime}$, gives a calibration of the micrometer scale in units (microns, millionths of an inch, etc.) of length for measuring the displacement of white-light fringes from a chosen reference point on the gage-block surface.

The procedure for adjusting a gage block is explained with the aid of inserts in figure 2. In general, when the block is placed on its supports, the light reflected from its end will not reach the observer because of excessive angular deviation from the 
eyepiece. The block will appear in silhouette, as indicated in figure $2 \mathrm{~B}$. When the surface $\mathrm{G}_{1}$ is adjusted approximately normal to the light, fine fringes will usually be visible in the area covered by both images of it, as shown in figure $2 \mathrm{C}$. The images of the two parts of $\mathrm{G}_{1}$ are made to coincide by rotating $\mathrm{G}$ about the center line of figure $2 \mathrm{~A}$, causing the image to change from that of figure $2 \mathrm{C}$ to figure 2D. A lateral motion, without rotation, will then change the image from that shown in figure $2 \mathrm{D}$ to figure $2 \mathrm{E}$. A further small rotation of $\mathrm{G}$ about an axis normal to figure $2 \mathrm{~A}$ brings $\mathrm{G}_{1}$ normal to the light and the fringes on $\mathrm{G}_{1}$ will appear horizontal and parallel to the background fringes as shown in figure $2 \mathrm{~F}$. The observer then moves to position $\mathrm{S}_{2}{ }^{\prime}$ and observes the set of fringes shown in figure $2 \mathrm{G}$. The angle between these two sets of fringes corresponds to the angle between surfaces $G_{1}$ and $G_{2}$.

A photograph of the long-block interferometer is shown in figure 3 . The base of the instrument is designed for rigidity so as to avoid flexure. Rigidity is quite important. The two prisms are mounted on rigid tables at each end of the base. Three screws ( $D_{1}$ and two others not shown) permit raising and lowering of prism $\mathrm{P}_{2}$. They also permit rotation or tilting of this prism about any chosen horizontal axis. The prism housings are fastened to the table tops by means of large-headed screws, in oversize holes, that permit lateral adjustments of the prisms relative to each other. Two screws, $\mathrm{D}_{2}$ and another concealed by the housing of prism $\mathrm{P}_{2}$, permit small rotations of this prism about a vertical axis by applying lateral torques to the legs of the table. Similarly, screws $\mathrm{D}_{3}$ and $\mathrm{D}_{4}$ permit rotary adjustments of the other prism housing.

The adjustments described above permit the alinement of the two prisms. This adjustment is critical, rather difficult to attain, but when once obtained is very stable. The final adjustments are executed while observing interference fringes produced by lens $\mathrm{L}_{2}^{\prime}$, in one of the many images of the source $\mathrm{S}_{2}$. This author uses a pinhole source of approximately $1-\mathrm{mm}$ diam and a short-focus lens to observe its image at $\mathrm{S}_{2}{ }^{\prime}$. There are two sets of fringes, superimposed upon each other, in the proper image to be used. When these two sets of fringes are horizontal and very broad, fringes can be seen with the two eyepieces when focused on the pinhole. These fringes, in white light, are horizontal with the zero order in the center of the field.

The supports on which the long blocks rest are located at the Airy points so as to reduce changes in the angle between $\mathrm{G}_{1}$ and $\mathrm{G}_{2}$ due to gravitational distortion. ${ }^{5}$ These supports rest on an adjustable plate which, in turn, is supported at one end by two points and at the other by one point. This plate is adjustable at one end, laterally with screw $D_{5}$ and vertically with another screw $\mathrm{D}_{6}$ which is concealed in figure 3.

\subsection{Short-Block Interferometer}

The optics of the short-block interferometer are shown in figure 4 . The double-image prism, lenses light source, and viewing position in figure $4 \mathrm{~B}$ are identical to that of either end of the long-block instrument described above. A reflecting prism, $\mathrm{P}_{\mathrm{R}}$ in figure 4, replaces one of the prism assemblies of the long-block instrument. Also, the optical axis of the instrument is vertical instead of horizontal. Figure 5 is a photograph of the short-block interferometer. Figure $4 \mathrm{~A}$ may be considered a section through the center of $4 \mathrm{~B}$, coincident with the dividing plane of prism $\mathrm{P}$. The indicated rays, 1 and 2 in figure $4 \mathrm{~A}$, however, do not lie in this plane. Their positions relative to it are indicated in $4 \mathrm{E}$, which is a vertical view through $4 \mathrm{~B}$. The two surfaces, $\mathrm{G}_{1}$ and $\mathrm{G}_{2}$, of the gage block (figs. $4 \mathrm{~A}$ and $4 \mathrm{D}$ ) appear as $\mathrm{G}$ and $\mathrm{G}^{\prime}$ in figure $4 \mathrm{E} . \mathrm{G}^{\prime}{ }_{2}$ in figure $4 \mathrm{~A}$ is an image of $\mathrm{G}_{2}$ as seen by light reflected from the right-angle prism, $P_{R}$.

${ }^{5}$ F. H. Rolt, Gauges and fine measurements II, 340 (Macmillan and Co., 1929).

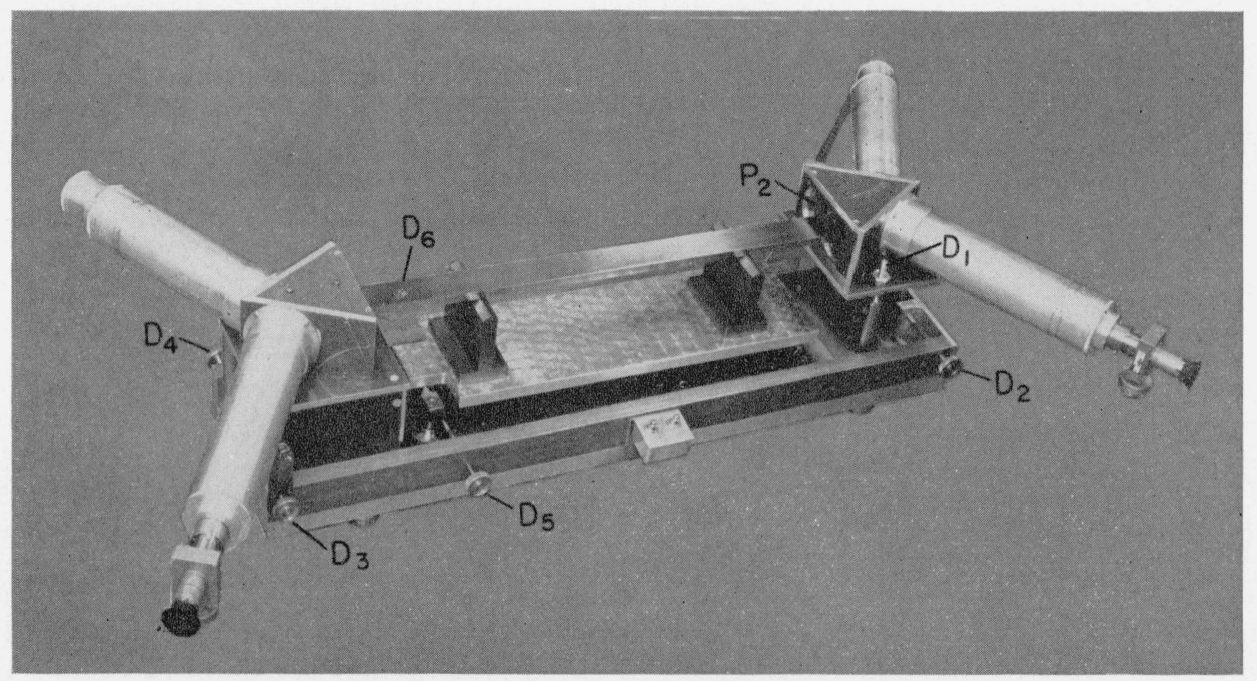

FIguRE 3. Photograph of the long-block interferometer. 


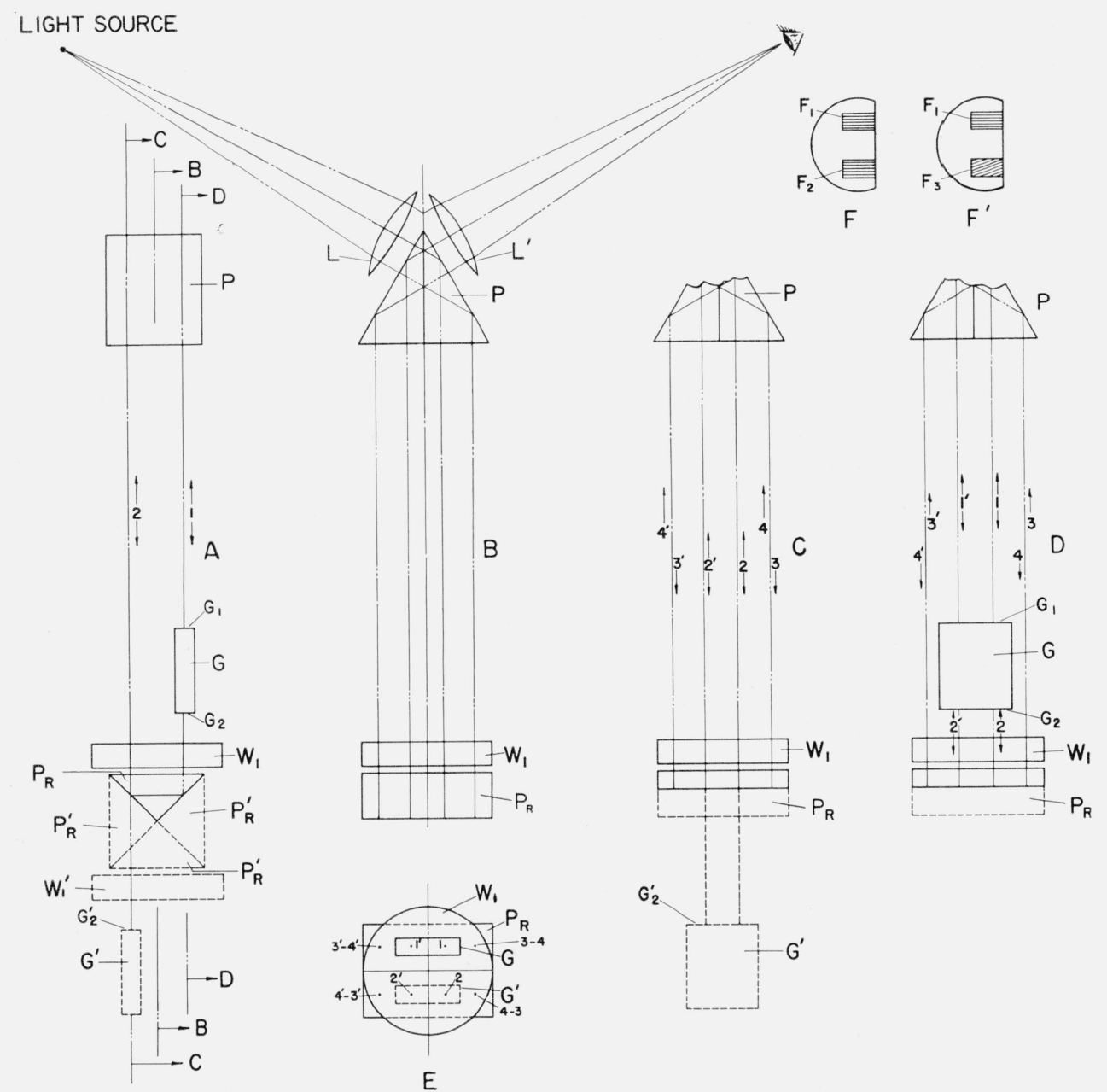

FiguRE 4. Optics of the interferometer for testing parallelism of short blocks.

Figures B, C, and D are sections through A. Figure $\mathrm{E}$ is a vertical view. Figures $\mathrm{F}$ and $\mathrm{F}^{\prime}$ represent interference fringes for a parallel and a nonparallel block, respectively.

A plane optical wedge, $W_{1}$, and its image, $\mathrm{W}^{\prime}{ }_{1}$, (in figure $4 \mathrm{~A}$ ) are shown between $\mathrm{G}$ and its image $\mathrm{G}^{\prime}$. Figures $4 \mathrm{~B}, 4 \mathrm{C}$, and $4 \mathrm{D}$ are sections through $4 \mathrm{~A}$, along the lines indicated. The gage block is not located in the center as was the case in the instrument described above.

The two component rays of light, 1 and $1^{\prime}$ (figs. $4 \mathrm{~A}$ and $4 \mathrm{D})$, are caused to reflect normally from $\mathrm{G}_{1}$ by adjusting $\mathrm{G}$ with a leveling screw. They return into $\mathrm{P}$ where they recombine to produce the interference fringes, $\mathrm{F}_{1}$, shown in figure $4 \mathrm{~F}$. The two component rays, 2 and $2^{\prime}$ (figs. $4 \mathrm{~A}$ and $4 \mathrm{C}$ ) are transmitted downward through the optical wedge, $\mathrm{W}_{1}$, suffer two internal reflections in $\mathrm{P}_{\mathrm{R}}$, and if $\mathrm{P}_{\mathrm{R}}$ is properly adjusted, return upward and parallel to their directions of incidence through $W_{1}$ to $\mathrm{G}_{2}$.

The wedge, $W_{1}$, is adjusted initially by rotation so that its thickness is constant at all points in either of the planes B, C, and D. When in this neutral position it does not affect the interference fringes because of compensation in each pair of component beams that pass through it. The function of $W_{1}$ will be explained later.
In order to measure the angle between $\mathrm{G}_{1}$ and $\mathrm{G}_{2}$, the deviation of the light by $\mathrm{P}_{\mathrm{R}}$ toward or from the dividing plane of $\mathrm{P}$ must either be reduced to zero or its effect eliminated by measuring the observed angle for two orientations of $G$, which are $180^{\circ}$ apart. The light that is not intercepted by G forms an interference pattern of uniform tint (or color) that fills the background about and between the two images of the gage block, shown in figure $4 \mathrm{~F}$ or $4 \mathrm{~F}^{\prime}$. Figures $4 \mathrm{~F}$ and $4 \mathrm{~F}^{\prime}$ represent the conditions observed when the ends of the block are parallel and nonparallel, respectively. A typical pair of component rays, which form this interference pattern, is indicated by 3 and $3^{\prime}$. They travel downward in figure $4 \mathrm{C}$ and upward in figure $4 \mathrm{D}$. If the right-angle edge of $P_{R}$ is normal to the dividing plane of $\mathrm{P}$, the pair of rays 3 and $3^{\prime}$ in figure $4 \mathrm{C}$ can be made to return in planes that are parallel to the dividing plane, by rotating $\mathrm{P}_{\mathrm{R}}$ about an axis parallel to the plane of $4 \mathrm{~A}$ and normal to the incident light. This condition is attained when the background fringe is infinitely broad. The direction of the background fringes, when not infinitely broad, 


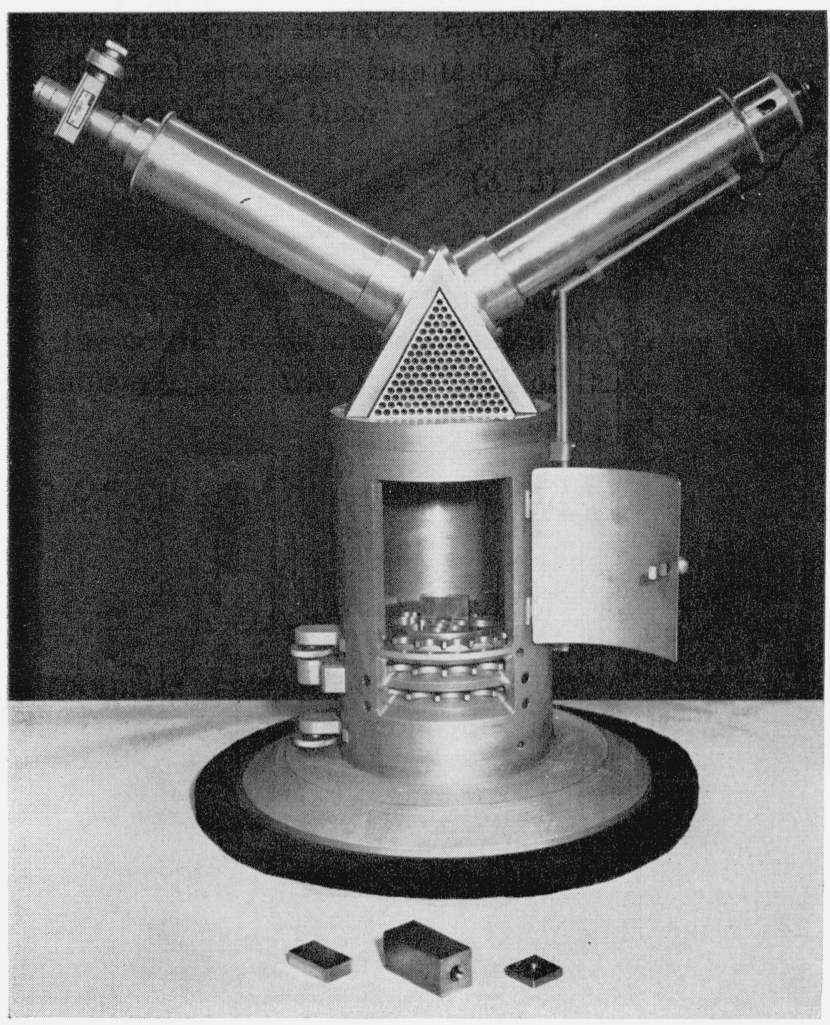

Figure 5. Photograph of the short-block interferometer.

remain parallel to the dividing plane, because for each pair of component rays, such as 3 and $3^{\prime}$, there is a corresponding pair, 4 and $4^{\prime}$, that travel identical paths but in opposite directions. The optical path differences are, therefore, equal to each other and also equal to that for any other pair of component rays in the plane of $4 \mathrm{C}$ and $4 \mathrm{D}$. The order of interference along the dividing plane corresponds to the optical path difference that was introduced into the double-image prism by the built-in wedges at the point that corresponds to the point of intersection of the right-angle edge of $P_{R}$ and the dividing plane of $\mathrm{P}$. This point is located in the center of figure $4 \mathrm{E}$.

In general, due to the inherent error of judging when the background fringes are infinitely broad and to imperfections in the optical elements, a more precise method of evaluating the wedge between $\mathrm{G}_{1}$ and $\mathrm{G}_{2}$ is to measure the wedge for two positions that differ by $180^{\circ}$. If the background fringes are unaltered, the instrumental errors will be equal for the two positions and the value of the wedge unchanged except in sign. Consequently, the albebraic difference yields twice the value of the wedge.

There are three ways that one might evaluate the wedge between $\mathrm{G}_{1}$ and $\mathrm{G}_{2}$. The first is to rotate $\mathrm{P}_{\mathrm{R}}$ until the order of interference at points $\mathrm{C}$ and $\mathrm{E}$ (fig. $6 \mathrm{~A}$ ) are equal; then rotate the gage block until the orders at $A$ and $B$ are equal; and finally, observe the difference in order of interference at points $\mathrm{F}$ and $\mathrm{H}$. The second method is: After performing the above operations, instead of reading the order difference between $\mathrm{F}$ and $\mathrm{H}$, re-

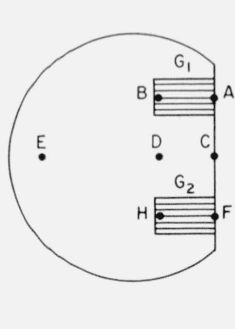

A
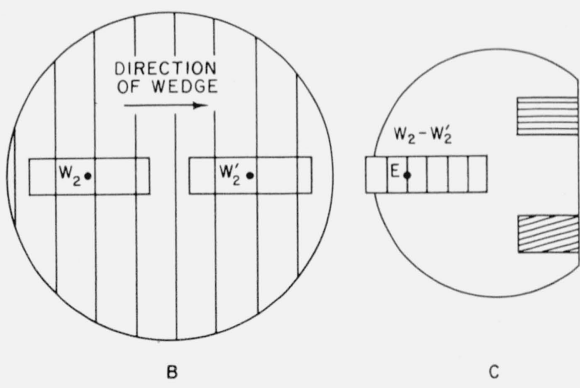

FIGURE 6. Figures $A$ and $C$ represent the positions of reference points relative to the different sets of interference fringes.

Figure B shows the wedge from which the two smaller wedges, $\mathrm{W}^{2}$ and $\mathrm{W}^{2}$, were cut and the difference in optical thickness between them.

duce this order difference to zero by rotating $W_{1}$ and read the resultant change on a scale attached to $\mathrm{W}_{1}$, figure $4 \mathrm{E}$. This scale may be calibrated with monochromatic light and the units may be radians, degrees, or the corresponding variation in height of the block. A third method is to leave the wedge in its neutral position, adjust $\mathrm{G}$ so that the orders of interference of $A$ and $B$ are equal, and change the order at $\mathrm{H}$ to equal that at $\mathrm{F}$ by rotating $P_{R}$ about an axis normal to the incident light and parallel to the plane of figure $4 \mathrm{~A}$. The order of interference between two points such as $\mathrm{C}$ and $\mathrm{D}$ (fig. 6A) after rotating $\mathrm{P}_{\mathrm{R}}$ will be equal to one-half of that between $\mathrm{F}$ and $\mathrm{H}$ before this rotation was performed. By choosing a point, such as $\mathrm{E}$ in figure 6 , such that $\mathrm{CE}$ equals $\mathrm{K}$ times $\mathrm{CD}$, the order difference between $\mathrm{C}$ and $\mathrm{E}$ will be $\mathrm{K}$ times that between $\mathrm{C}$ and $\mathrm{D}$.

When using this last method for testing gages that are almost parallel, the angle between $\mathrm{G}_{1}$ and $\mathrm{G}_{2}$ will be small and the background fringes will be too broad for reading fractions of fringes. To eliminate this difficulty, an optical wedge, illustrated in figure $6 \mathrm{~B}$, is constructed and from it two sections $\mathrm{W}_{2}$ and $\mathrm{W}_{2}^{\prime}$ are cut and placed on $\mathrm{P}_{\mathrm{R}}$ as shown in figure 7 . The wedges $W_{2}$ and $W_{2}^{\prime}$ are equal, but when placed in the position shown, the effect is to narrow the background fringes seen through them. The results are illustrated in figure $6 \mathrm{C}$. The difference in thickness of $W_{2}$ and $W_{2}^{\prime}$ at a selected reference point $\mathrm{E}$ (fig. 6C) is determined by the choice of the corresponding positions on the plate from which they were cut. This difference in thickness is chosen so as to cause the zero order of interference to pass through the chosen point when the background fringes about $\mathrm{W}_{2}-\mathrm{W}_{2}^{\prime}$ are infinitely broad.

If the angles of wedges $\mathrm{W}_{2}$ and $\mathrm{W}_{2}{ }^{\prime}$ are properly chosen, the width of the fringes seen through them will be most favorable for measuring the fractional parts of fringes. Also, the position of the zeroorder fringe, relative to point $\mathrm{E}$, may be calibrated to read directly the angle between the ends of the gage blocks.

The recommended procedure for measuring a block is: (1) Adjust the two sets of fringes seen on the ends of the block so that they are perpendicular to the dividing plane, as in figure $4 \mathrm{~F}$; (2) note the position 


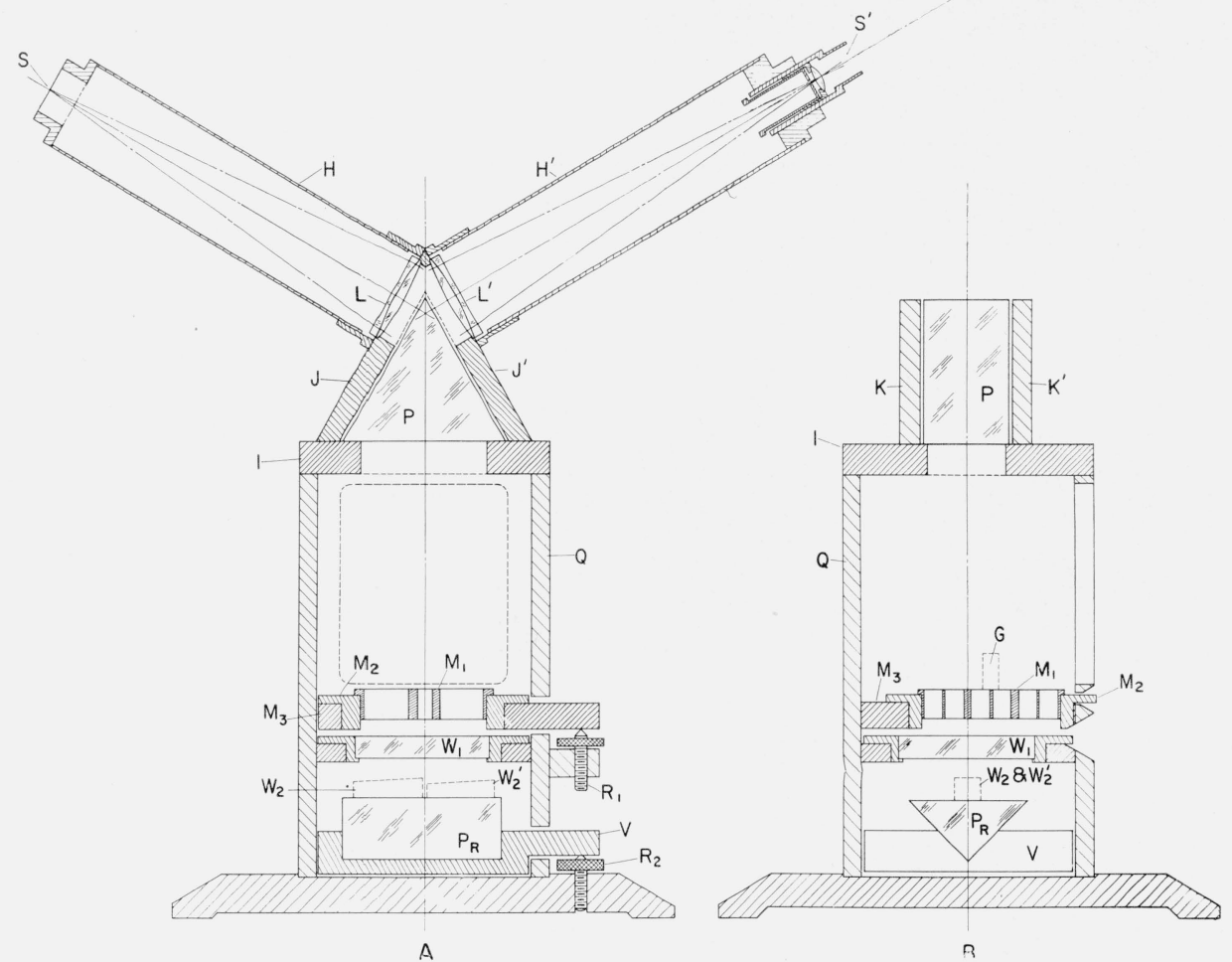

FiguRE 7. Two vertical sections, at right angles to each other, through the short-block interferometer.

of the zero-order fringe (or absolute order at $\mathrm{E}$, fig. $6 \mathrm{C})$; (3) rotate the block $180^{\circ}$ about a vertical axis through its center; (4) readjust the fringes to restore the condition of (1) above; (5) again note the position of the zero-order fringe (or absolute order at $\mathrm{E}) ;(6)$ the difference in the two observed orders at E, or positions of the zero-order fringe, multiplied by the constant $\mathrm{K}$, described above, is a measure of the angle between the ends of the block.

Figure 7 shows two vertical sections through the center of the short-block interferometer that are mutually perpendicular to and through the centers of each other. A pinhole, S, illuminated either with monochromatic or polychromatic light from outside the tube $H$, serves as source. The position of the pinhole is adjustable in the focal plane of the collimator lens, L, and the collimator tube is adjustable in length. The prism, P, rests on a thick plate, I, to which is fastened the lens-holding plates, $J$ and $J^{\prime}$. The plates, $\mathrm{K}$ and $\mathrm{K}^{\prime}$, which cover the ends of $\mathrm{P}$, are not fastened to $\mathrm{J}$ or $\mathrm{J}^{\prime}$. Consequently, small stresses applied to tube $\mathrm{H}^{\prime}$ while adjusting the eyepiece or manipulating a micrometer in it, are not transmitted to $\mathrm{P}$.

The material of the instrument, except for the collimator, the eye-piece tubes and optical elements, is made of steel. Steel was chosen because its expansivity approximates that of the glass elements more nearly than other usable materials.
The gage block, $\mathrm{G}$, rests on a rotatable plate, $\mathrm{M}_{1}$, that is perforated so as to transmit the required parts of the light beams used for making measurements. See also figure 8 for a vertical view of $M_{1}$ and its supporting parts. The plate, $\mathrm{M}_{1}$, rotates in an annulus ring, $\mathrm{M}_{2}$, which in turn is rotatable, from outside the instrument, in another annulus, $\mathrm{M}_{3}$. The aperture in $\mathrm{M}_{3}$ has its center displaced from the center of the instrument in a direction parallel to the dividing plane of $\mathrm{P}$ and by an amount equal to one-half the horizontal separation between the centers of the gage block, $\mathrm{G}_{1}$, and its image, $\mathrm{G}^{\prime}$. The gage block rests on the center of $\mathrm{M}_{1}$ (see fig. $6 \mathrm{~B}$ ). Accurate placement of the block is facilitated by stops.

The eccentric annulus, $\mathrm{M}_{3}$, has an arm that projects through the wall, Q, of the instrument and is supported by this arm at one of its three supporting points by an adjusting screw, $R_{1}$. The other two supports for $\mathrm{M}_{3}$ are steel balls, $\mathrm{T}_{1}$ and $\mathrm{T}_{2}$, (fig. 8B), which are held in conical holes by means of two screws, $\mathrm{U}_{1}$ and $\mathrm{U}_{2}$, respectively. The ends of these screws have eccentric conical depressions that permit a limited amount of rotation of $\mathrm{M}_{3}$ about an axis normal to the dividing plane of $\mathrm{P}$. The screw $\mathrm{R}_{1}$ permits fine adjustment of $\mathrm{M}_{2}$, and consequently the gage block, which it supports, about a horizontal axis parallel to the dividing plane of $\mathrm{P}$. A similar pair of balls, screws, and the adjustable screw $R_{2}$, permits rotation of $\mathrm{P}_{\mathrm{R}}$ about two axes parallel to those used for adjusting $\mathrm{M}_{3}$. 

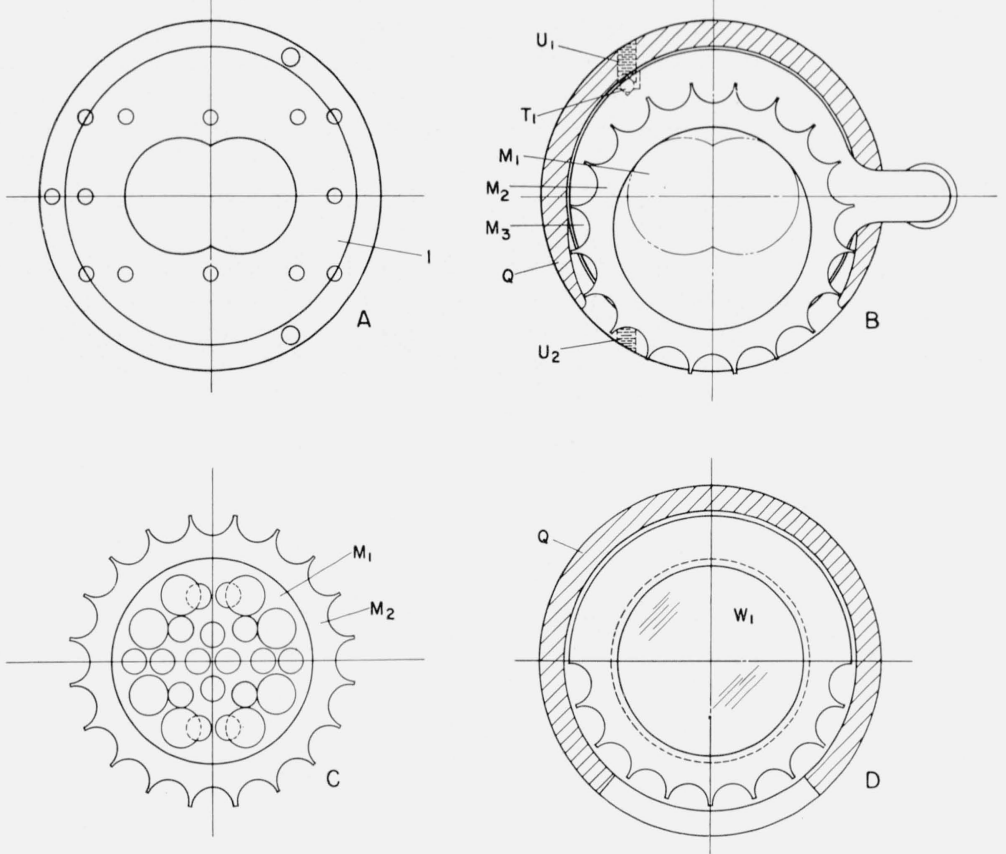

Figure 8. Several views showing individual parts of the short-block interferometer.

If the refracting edge of the optical wedge, $W_{1}$, is made perpendicular to the dividing plane of $\mathrm{P}$ (i. e., parallel to the plane of fig. 7B), each pair of component rays will traverse this plate at points of equal thickness. Consequently, $\mathrm{W}_{1}$ in this orientation, does not affect the fringes of interference. It does, however, serve as a window, protecting the prism $P_{R}$ from the accumulation of dust. Other functions of $\mathrm{W}_{1}$ will be discussed later.

The prism, $\mathrm{P}$, is centered over the aperture in plate $\mathrm{I}$ (figs. $7 \mathrm{~A}$ and $8 \mathrm{~A}$ ) with its ends parallel to plates $\mathrm{K}$ and $\mathrm{K}^{\prime}$ (fig. 7B). Using the base surface of $\mathrm{P}$ as a plane mirror, the pinhole aperture $\mathrm{S}$ is located in the focal plane of lens L by varying the length of tube $\mathrm{H}$ and at a point in this plane where the light will form an image of the pinhole upon itself. This places the light beams, after division at the dividing plane, normal to the prism base and, consequently, parallel to each other.

A gage block, whose end faces are parallel to each other, is placed on the center of plate $M_{1}$ with its lower surface parallel to the top surface of $\mathrm{M}_{1}$. When the surface of the block is adjusted parallel to the base of $\mathrm{P}$ by means of screws $\mathrm{R}_{1}, \mathrm{U}_{1}$, and $\mathrm{U}_{2}$, the light from $\mathrm{S}$ is reflected normally from the top surface of the block and observed at the eyepiece as interference fringes.

The parts of the light beams that are not intercepted by the block and its support $M_{1}$, traverse the optical wedge $W_{1}$, and enter the right-angle prism $P_{R}$. After two internal reflections in $\mathrm{P}_{\mathrm{R}}$, the light returns along a path that is symmetrical to its incident path with respect to the $90^{\circ}$ edge of $\mathrm{P}_{\mathrm{R}}$. The $90^{\circ}$ edge of $\mathrm{P}_{\mathrm{R}}$ is made normal to the dividing plane of $\mathrm{P}$ by means of screws in its support similar to $\mathrm{U}_{1}$ and $\mathrm{U}_{2}$ and the fine-adjusting screw $\mathrm{R}_{2}$ (fig. $7 \mathrm{~A}$ ). This light forms the background fringes used in the test, but no interference is observed until this prism edge is nearly normal to the dividing plane. When it is not normal the two images of this edge, formed by the two component light beams, intersect in the extension of the beam dividing plane. The prism $P_{R}$ is rotated until the two images coincide-perfection being attained when the background fringes have maximum contrast.

The above-mentioned contrast in the background fringes is not affected by screw, $R_{2}$, since it has no vertical rotational effect on the prism. Its effect is to change the width of the background fringes only. When the background fringes are made infinitely broad, the collimated beam of light returns toward $\mathrm{P}$ parallel to the incident beam - all rays having suffered a horizontal shift in $\mathrm{P}_{\mathrm{R}}$, as illustrated in figure $4 \mathrm{~A}$.

The holes in the gage-block support, $\mathrm{M}_{1}$ (fig. 8C), are so spaced that when $M_{1}$ is rotated to one of the four positions for which the rectangular sides of the block are either parallel or perpendicular to the dividing plane, all light that goes through $\mathrm{M}_{1}$ returns again through it. That is, the apertures in $\mathrm{M}_{1}$ are symmetrical both with respect to the dividing plane and to the $90^{\circ}$ edge of $\mathrm{P}_{\mathrm{R}}$. Parts of the beam (rays $3,3^{\prime}, 4$, and $4^{\prime}$ in fig. 4) will pass downward through $\mathrm{M}_{1}$ to $\mathrm{P}_{\mathrm{R}}$, shift horizontally in $\mathrm{P}_{\mathrm{R}}$, and pass upward through other apertures in $\mathrm{M}_{1}$ to $\mathrm{P}$. Other parts of the beam (rays 2 and $2^{\prime}$ in fig. 4) will pass downward through $\mathrm{M}_{1}$, shift horizontally in $\mathrm{P}_{\mathrm{R}}$, pass 
upward through $\mathrm{M}_{1}$ to the lower surface of the gage block, return through $\mathrm{M}_{1}$ to $\mathrm{P}_{\mathrm{R}}$ and again upward through $M_{1}$ to $P$. If the two end faces, $G_{1}$ and $G_{2}$, are parallel, the light will be incident on $\mathrm{G}_{1}$ and $\mathrm{G}_{2}$ at equal angles. When $\mathrm{G}_{1}$ is adjusted normal to the light, $\mathrm{G}_{2}$ will also be normal to it.

We have three sets of fringes, shown in figure $4 \mathrm{~F}^{\prime}$, to consider: (1) The set $\mathrm{F}_{1}$ is formed by light reflected from the top surface, $\mathrm{G}_{1}$, of the gage block; (2) the background fringe between and about $\mathrm{F}_{1}$ and $\mathrm{F}_{3}$, formed by light reflected from $\mathrm{P}_{\mathrm{R}}$ but not incident on the gage block; and (3) the set $\mathrm{F}_{3}$ formed by light reflected from $P_{R}$ to $G_{2}$ and back through $\mathrm{P}_{\mathrm{R}}$. The direction or orientation of $\mathrm{F}_{1}$ determines the angle between $\mathrm{G}_{1}$ and the incident wavefront; the width of the background fringes determine the direction between the incident and reflected beams to and from $\mathrm{P}_{\mathrm{R}}$; and the orientation of $\mathrm{F}_{3}$ determines the angle between $\mathrm{G}_{2}$ and the wavefront that is reflected from it.
The set of fringes, $F_{1}$, is adjusted by means of $R_{1}$ normal to the dividing plane of $\mathrm{P}$, for which condition $\mathrm{G}_{1}$ is normal to the incident light. The background fringe is made infinitely broad, for which condition the light beams returning from $P_{R}$ are parallel to the incident beams. If the two surfaces of $\mathrm{G}$ are parallel, the set of fringes appearing on $\mathrm{G}_{2}$ will be parallel to those on $\mathrm{G}_{1}$ and indicated as the set $\mathrm{F}_{2}$ in figure $4 \mathrm{~F}$. If $\mathrm{G}_{1}$ and $\mathrm{G}_{2}$ are not parallel, the fringes seen on them and indicated as $\mathrm{F}_{1}$ and $\mathrm{F}_{3}$ in figure $4 \mathrm{~F}^{\prime}$ will not be parallel to each other. The angle between these two sets of fringes is a measure of the angle between the two ends of the gage block.

Washington, May 5, 1958. 